\title{
El riesgo de eventos cardiovasculares mortales al suspender dosis bajas de aspirina por sangrado gastroduodenal superaría el de resangrado
}

Mortal cardiovascular risk after discontinuation aspirin low doses because gastroduodenal bleeding seems to exceed re-bleeding risk

\section{Objetivos}

Comparar el riesgo de recurrencia de eventos vasculares al suspender aspirina por úlceras sangrantes con el riesgo de resangrado.

\section{Diseño, lugar y pacientes}

Estudio aleatorizado, controlado y doble ciego; realizado en el Centro Endoscópico del Hospital Universitario Príncipe de Gales, Hong Kong, China.

Se seleccionaron pacientes con evidencia endoscópica de sangrado activo (úlcera con sangrado activo, vaso sangrante visible o coágulo adherido visible) derivados por presentar melena o hematemesis, que vinieran tomando dosis diarias de aspirina menores de $325 \mathrm{mg}$ o doble antiagregación indicada como prevención primaria o secundaria de enfermedad vascular, y que hubieran tenido buena respuesta al tratamiento endoscópico. Fueron excluidos los pacientes en quienes el procedimiento
Sung J, y col. Annals of Internal Medicine. Jan 2010; 152:1-9 endoscópico no había sido exitoso o la etiología del sangrado no había podido ser adjudicada solo a aspirina.

Casi el $80 \%$ de los pacientes habían sufrido un evento cardio o cerebrovascular (prevención secundaria).

\section{Intervención y medición de resultados principales}

Luego del tratamiento endoscópico, todos los pacientes recibieron una carga de $80 \mathrm{mg}$ de pantoprazol endovenoso seguida de $8 \mathrm{mg} / \mathrm{h}$ durante $72 \mathrm{~h}$, y fueron aleatorizados para recibir durante ocho semanas: a) $40 \mathrm{mg} / \mathrm{día}$ de pantoprazol más $80 \mathrm{mg}$ de aspirina; b) $40 \mathrm{mg} /$ día de pantoprazol más placebo.

El resultado principal fue la recurrencia de úlcera sangrante a los 30 días, y el secundario, las causas de muerte durante las primeras ocho semanas.

Resultados principales

Se describen en la tabla 1.

Tabla 1: Incidencia de los eventos evaluados según la rama de tratamiento asignado.

\begin{tabular}{|c|c|c|c|c|c|c|c|}
\hline \multirow[t]{2}{*}{ Evento evaluado } & \multicolumn{2}{|c|}{ Aspirina $n=78$} & \multicolumn{2}{|c|}{ Placebo } & \multirow[t]{2}{*}{ Riesgo relativo } & \multirow{2}{*}{$\begin{array}{c}\text { Reducción de riesgo } \\
\text { absoluto }\end{array}$} & \multirow[t]{2}{*}{ NNT } \\
\hline & n (\%) & $\mathbf{R A}$ & n (\%) & $\mathbf{R A}$ & & & \\
\hline Sospecha de resangrado & $13(16,80)$ & 0,17 & $9(12,00)$ & 0,11 & 1,44 & 0,05 & 19,6 \\
\hline $\begin{array}{l}\text { Confirmación endoscópica de } \\
\text { resangrado }\end{array}$ & $8(10,30)$ & 0,10 & $4(5,40)$ & 0,05 & 2 & 0,05 & 19,6 \\
\hline $\begin{array}{l}\text { Mortalidad por eventos } \\
\text { cardiovasculares }\end{array}$ & $1(1,30)$ & 0,01 & $5(6,40)$ & 0,06 & 0,2 & 0,05 & 19,5 \\
\hline
\end{tabular}

$\mathrm{RR}$ : riesgo relativo RA: riesgo absoluto NNT: número necesario a tratar.

\section{Conclusiones}

Continuar el tratamiento con aspirina luego del tratamiento endoscópico de úlceras sangrantes aumentaría el riesgo de resangrado pero reduciría la tasa de mortalidad.
Palabras claves: aspirina, hemorragia digestiva, prevención cardiovascular. Key words: aspirine, gastrointestinal bleeding, cardiovascular prevention. Fuente de financiamiento: Instituto de Enfermedades Digestivas. Universidad de Hong Kong. Conflicto de interés: Altana fue el proveedor de Pantoprazol, pero no participó en el diseño, recolección de datos ni análisis estadístico.

\section{Comentario}

Existe evidencia ${ }^{1}$ que avala la utilización de aspirina en prevención cardiovascular. También es conocida la participación de los antiinflamatorios no esteroides (AINE) en la fisiopatología de la úlcera gastroduodenal'.

Si bien en este estudio no se realizaron análisis de subgrupos (prevención primaria y secundaria), dado que el $80 \%$ de los pacientes incluidos recibían aspirina como prevención secundaria de la enfermedad cardiovascular, sus resultados sugieren que la estrategia de continuar con aspirina a dosis bajas sumada al uso de inhibidores de la bomba de protones aumenta el riesgo de resangrado de las úlceras gastroduodenales, disminuyendo sin embargo la mortalidad, principalmente la asociada a eventos vasculares.
Algunas de las limitaciones que presenta este estudio son el escaso número de pacientes enrolados y el haber evaluado solamente el uso de aspirina en dosis de $80 \mathrm{mg}$.

\section{Conclusiones de la comentadora}

Todavía quedaría pendiente determinar si la presencia de algunos potenciales predictores de resangrado (presencia de $\mathrm{H}$. pylori, patología gástrica asociada, etc.) ayudarían a estratificar el riesgo y contribuirían a decidir a continuar o no el tratamiento con aspirina en los pacientes que están siendo tratados por una úlcera gastroduodenal sangrante.

María Eugenia Barile [ Departamento de Medicina Interna, Servicio de Clínica Médica, Hospital Nuestra Señora de la Misericordia, Córdoba. Argentina. eugeniabarile@gmail.com ]

Recibido el 10/08/10 y aceptado el 04/04/11

Barile M. El riesgo de eventos vasculares mortales al suspender dosis bajas de aspirina por sangrado gástrico supera el riesgo de resangrado. Evid Act Pract Amb. 14(2).46. Abr-Jun. 2011. Comentado de: Sung J, y col. Continuation of low dosis aspirin therapy in peptic ulcer bleedding. Annals of Internal Medicine. Jan 2010; 152:1-9. PMID: 19949136.

Referencias

1. Patrono C y col. Low-Dose Aspirin for the Prevention of Atherothrombosis. N Engl J Med 2005; 353:2373-2383.

2 Gobierno Federal de México. Guía de práctica clínica. Diagnóstico y tratamiento de la úlcera péptica complicada: Conceptos básicos. Evidencia y recomendaciones. 2009.

3 Leontiadis G y col. Systematic reviews of the clinical effectiveness and costo-effectiveness of protom pump inhibitor in acute upper gastro intestinal bleeding. Health Technology Assessment 2007: Vol 11: number 51.

4 Lau J y col. Omeprazole before Endoscopy in Patients with Gastrointestinal Bleeding. N Engl J Med 2007; 356; 16: 1631-1640.

5 Sreedharan A y col. Proton pump inhibitor treatment initiated prior to endoscopic diagnosis in upper gastrointestinal bleeding. Cochrane Database of Systematic Reviews 2010 , Issue 7. Art. No.: CD005415. DOI: 10.1002/14651858.CD005415.pub3

6 Catella-Lawson F y col. Cyclooxygenase inhibitors and the antiplatelet effects of aspirin. N Engl J Med 2001; 345:1809-17.

7 Cryer B. Reducing the Risks of Gastrointestinal Bleeding with Antiplatelet Therapies. N Engl J Med 2005; 352:287-289. 